Check for updates

Cite this: RSC Adv., 2019, 9, 15957

\title{
Effects of different electrolytes and film thicknesses on structural and thermoelectric properties of electropolymerized poly(3,4- ethylenedioxythiophene) films $\uparrow$
}

\begin{abstract}
Yuhei Seki, Momoko Takahashi and Masayuki Takashiri (iD *
The effects of the type of electrolyte and film thickness on the structural and thermoelectric properties of poly(3,4-ethylenedioxythiophene) (PEDOT) thin films on indium-tin-oxide (ITO) substrates prepared using electropolymerization were investigated. Two electrolytes were prepared using two different solvents: a water/methanol solvent (protic solvent) and acetonitrile (aprotic solvent) with 3,4ethylenedioxythiophene (EDOT) and $\mathrm{LiCF}_{3} \mathrm{SO}_{3}$, typically included in electrolytes as dopants. The electrochemical properties of the two electrolytes were analyzed; it was found that the polymerization process for EDOT on an ITO substrate varied based on the electrolyte used. When the electropolymerization time was increased, the surface morphology of the PEDOT films prepared using the water/methanol solvent appeared to contain grains approximately $100 \mathrm{~nm}$ in size whereas the PEDOT films prepared using acetonitrile appeared to contain aggregated grains connected by polymeric networks. Even though there were differences in the surface morphology and chemical bonds determined using Fourier-transform infrared spectroscopy/attenuated total reflectance analysis, the thermoelectric properties were strongly dependent on the film thickness and were only weakly dependent on the type of electrolyte used. The highest power factor was $41.3 \mu \mathrm{W}\left(\mathrm{m}^{-1} \mathrm{~K}^{-2}\right)$ for a PEDOT

film with a thickness of $0.5 \mu \mathrm{m}$ prepared using the water/methanol solvent electrolyte.
\end{abstract}

Received 26th March 2019 Accepted 13th May 2019

DOI: 10.1039/c9ra02310k

rsc.li/rsc-advances

\section{Introduction}

Organic conducting polymers, including polyaniline, polypyrrole, polythiophene, and their derivatives, have attracted considerable attention because of their broad application in a variety of devices such as energy storage systems, ${ }^{1-3}$ sensors and actuators, ${ }^{4-6}$ solar cells, ${ }^{7,8}$ and thermoelectric generators. ${ }^{9-12}$ Amongst conducting polymers, the polythiophene derivative poly(3,4-ethylenedioxythiophene) (PEDOT) or $\operatorname{poly}(3,4-$ ethylenedioxythiophene):poly(styrenesulfonate) (PEDOT:PSS) is considered to be one of the best conducting polymers currently available in terms of conductivity, stability, fineprocessability, and flexibility. ${ }^{13}$

In particular, these characteristics of PEDOT are suitable for its use in thin-film thermoelectric generators. ${ }^{14,15}$ Thin-film thermoelectric generators are composed of a number of thermoelectric thin films arranged in a pattern of strips and connected with thin metal electrodes. ${ }^{16-19}$ Electric power is produced using heat flow across a temperature gradient

Department of Materials Science, Tokai University, 4-1-1 Kitakaname, Hiratsuka, Kanagawa 259-1292, Japan. E-mail: takashiri@tokai-u.jp

$\dagger$ Electronic supplementary information (ESI) available. See DOI: $10.1039 / \mathrm{c} 9 \mathrm{ra} 02310 \mathrm{k}$ between both ends of the thin films. The performance of thermoelectric generators is proportional to their power factor (PF) and the dimensionless figure-of-merit $Z T$. PF and $Z T$ are defined as $\mathrm{PF}=\sigma S^{2}$ and $Z T=\sigma S^{2} T / \kappa$, respectively, where $\sigma$ is the electrical conductivity, $S$ is the Seebeck coefficient, $T$ is the absolute temperature, and $\kappa$ is the thermal conductivity. Therefore, higher electrical conductivity in the PEDOT contributes to higher performance in the thermoelectric generators that incorporate it. The output voltage $V(V=n S \Delta T)$ of the generator depends on the Seebeck coefficient $S$, the temperature difference $\Delta T$, and the number of thin-film strips used in the pattern $n$. The fine-processability of PEDOT contributes to an increase in the output voltage by increasing the number of thin-film strips that can be incorporated into the pattern. The stability of the PEDOT performance is of benefit when the generators are repeatedly subjected to a changing temperature difference. The flexibility of PEDOT facilitates attachment of the generators to nonplanar surfaces such as pipes or human bodies. ${ }^{20}$

PEDOT thin films can be synthesized both in organic and aqueous solutions using electrochemical methods..$^{21-25}$ Electropolymerization is known to be a reliable electrochemical method for fabrication of organic thin films. ${ }^{26,27}$ Electropolymerization of PEDOT thin films in an organic solution has typically required the use of acetonitrile as the electrolyte 
solvent to polymerize the 3,4-ethylenedioxythiophene (EDOT) monomers. ${ }^{28-31}$ Although the electropolymerized PEDOT thin films prepared using acetonitrile, which is an aprotic solvent, exhibit high performance, acetonitrile is harmful to human health and the environment. On the other hand, the preparation of electropolymerized PEDOT thin films using an aqueous solution is relatively environmentally friendly. However, forming PEDOT films in an aqueous solution is difficult because of the low water solubility of EDOT monomers and their high oxidation potential. In addition, the relatively strong nucleophilic reactivity of water molecules with the initially formed thienyl radical cations causes water decomposition instead of electropolymerization. ${ }^{32}$ To solve these problems, Sakmeche et al. used surfactant molecules such as sodium dodecyl sulfate. $^{33}$ Furthermore, 3,4-ethylenedioxythiophene-methanol (EDTM), which is a water-soluble monomer, was synthesized from EDOT and methanol using a relatively simple process. ${ }^{34,35}$ Therefore, electropolymerization of PEDOT thin films can be performed using an electrolyte with a protic solvent mixture composed of methanol and water. Furthermore, it is necessary to investigate the optimum film thickness for PEDOT films because organic conducting polymers change in electrical conductivity with increasing film thickness. ${ }^{36,37}$

In this study, PEDOT thin films were fabricated using electropolymerization on indium-tin-oxide (ITO) substrates with methanol/water and acetonitrile as the electrolytes. The electropolymerization time was changed to increase the film thickness. The surface morphology and chemical structures of the PEDOT thin films prepared using different electrolytes and electropolymerization times were analyzed and their thermoelectric properties at temperatures near to $300 \mathrm{~K}$ were measured. The changing relationship between the structural and thermoelectric properties of the PEDOT thin films caused by changes in the electrolytes and electropolymerization times linked to the film thickness was analyzed.

\section{Experimental}

Pure grade EDOT (purity $>97 \%$ ) was purchased from FUJIFILM Wako Pure Chemical Industries, Ltd. (Japan). Pure grade lithium-trifluoromethanesulfonate $\left(\mathrm{LiCF}_{3} \mathrm{SO}_{3}\right)$, pure grade methanol (purity $>99.8 \%$ ), and pure grade acetonitrile (purity $>$ 99.5\%) were purchased from FUJIFILM Wako Pure Chemical Industries, Ltd. (Japan). All chemicals were used without further purification. ITO film $(0.3 \mu \mathrm{m}$ thick $)$ on glass substrates of dimensions $50 \mathrm{~mm} \times 20 \mathrm{~mm} \times 1.1 \mathrm{~mm}$ thick were purchased from the Geomatec Corporation (Japan).

Prior to the preparation of the PEDOT thin films, the oxidation and reduction behaviors in the electrolytes were measured using cyclic voltammetry (CV) performed with a standard three-electrode cell system. A platinum-coated $\mathrm{Ti}$ mesh was used as the counter electrode and an $\mathrm{Ag} / \mathrm{AgCl}$ (saturated $\mathrm{KCl}$ ) was used as the reference electrode. The surface areas of the working electrode and counter electrode were set to 5 and $10 \mathrm{~cm}^{2}$, respectively. Two types of organic solvents were prepared: methanol diluted with ion-exchanged water (ratio of $1: 1)$ and acetonitrile with EDOT (0.01 M) and $\mathrm{LiCF}_{3} \mathrm{SO}_{3}$ as dopants $(0.1 \mathrm{M})$ dispersed in the organic solvents $(100 \mathrm{ml})$. An automatic polarization system (Hokuto Denko HSV-110) was used to apply potentials within a range of $\pm 2.0 \mathrm{~V}$ at a scanning rate of $5,10,20$, or $30 \mathrm{mV} \mathrm{s}^{-1}$.

The PEDOT films incorporating the dopant were electropolymerized on ITO-coated glass, using the ITO coating as the working electrode in the standard three-electrode cell system. The system used for the electropolymerization of PEDOT films was the same system used for the CV measurements, with the exception of the incorporation of a potentiostat (Hokuto Denko HA-151B). The applied potential was set at a value of $1.52 \mathrm{~V}$ as determined by the results of the $\mathrm{CV}$ analysis, while the deposition time was varied from 10 to $480 \mathrm{~s}$. The resulting films were dried in air for approximately $24 \mathrm{~h}$. The film thickness was determined based on the difference between the mass of the initial substrate and that of the substrate covered with the PEDOT film. ${ }^{38}$

The surface morphologies of the samples were observed using field emission scanning electron microscopy (FE-SEM) (FE-SEM; Hitachi, S-4800). The chemical structures of the films were characterized using Fourier transform infrared spectroscopy with attenuated total reflectance (FT-IR/ATR; JASCO FT/IR-4200). The in-plane electrical conductivity $\sigma$ of the samples was measured at a temperature near $300 \mathrm{~K}$ using the four-point probe method (NAPSON RT-70V). The in-plane Seebeck coefficient $S$ was also measured at a temperature near $300 \mathrm{~K}$. One end of the thin film was connected to a heat sink and the other was connected to a heater. The Seebeck coefficient was determined as the ratio of the potential difference across the membrane to the temperature difference measured using two $0.1 \mathrm{~mm}$-diameter K-type thermocouples pressed against the membrane. The in-plane power factor $\sigma S^{2}$ was calculated from the measured electrical conductivity and Seebeck coefficient.

\section{Results and discussion}

\section{Electrochemical analyses of different electrolytes}

Fig. 1 shows the first set of cyclic voltammograms generated using different scanning rates for the two electrolytes: water/ methanol and acetonitrile. The onset potential was determined from the intersection of the tangents drawn at the baseline current and oxidation current slope in the cyclic voltammogram. ${ }^{39}$ A crossover was observed between the forward and reverse scans in the cyclic voltammogram called a "nucleation loop." This phenomenon was attributed to the initial stage in the nucleation process of the conductive polymer. ${ }^{40}$ In the cyclic voltammogram of the water/methanol electrolyte shown in Fig. 1(a), the value of the onset potential is approximately $1.14 \mathrm{~V}$ for all the scanning rates, while the nucleation loops increase as the scanning rate increased. This phenomenon indicated that the diffusion process to form the PEDOT film via oxidation of the EDOT monomer was influenced by the scanning rate. In Fig. 1(b), the cyclic voltammogram of the acetonitrile electrolyte exhibits almost identical curves for all scanning rates, indicating that the diffusion process that formed the PEDOT film did not depend on the scanning rate. The onset potential was approximately $1.32 \mathrm{~V}$, which was higher 

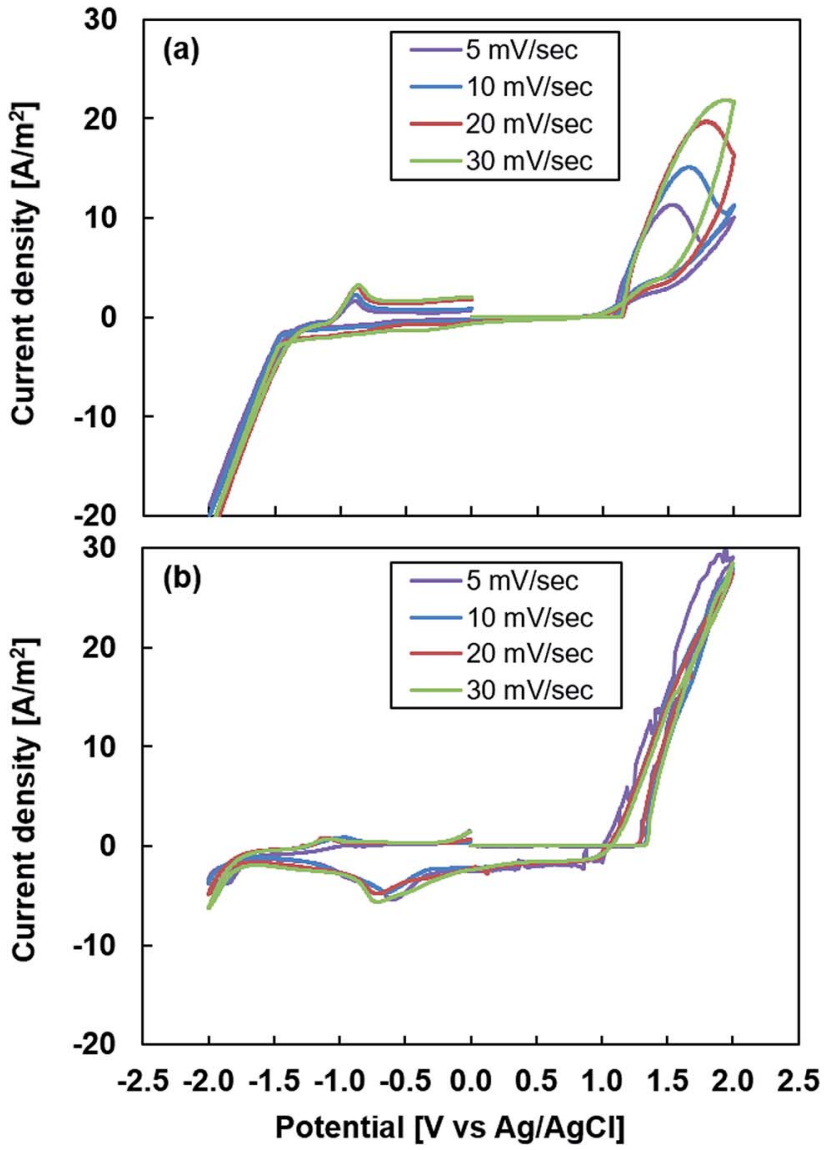

Fig. 1 First set of cyclic voltammometry experiments with different scanning rates performed during electropolymerization using (a) water/methanol electrolyte and (b) acetonitrile electrolyte.

than that of the water/methanol electrolyte because an aqueous medium facilitates polymerization. ${ }^{41}$ The discrepancies between oxidation potentials can be explained by the different solubilities of EDOT oligomers produced during the initial stages of electropolymerization. ${ }^{42}$ The influence of water on the polymerization rate of pyrrole, known as the "water effect", has already been reported by Zhou et al. who proposed that water impeded the acid-catalyzed formation of a pyrrole trimer, 2,20(2,5-pyrrolidinediyl)dipyrrole, and its further electropolymerization and passivation of the electrode through nonconductive deposits. ${ }^{43}$ It was proposed that EDOT followed an analogous electropolymerization mechanism; thus, it was influenced by the "water effect" in a manner similar to pyrrole. Except for the "water effect", the growth mechanism of PEDOT films in the two electrolytes is considered to be the same; the principal mechanism for the electropolymerization of PEDOT films is as follows. The initiation step involves the anodic oxidation of monomers to their radical cations in the vicinity of the anode. The radical cations then dimerize. After this step, the dimer is reoxidized and couples with another radical cation. Reoxidation follows, and the process continues with the formation of oligomer species. Once the chain length of the oligomers exceeds the solubility limit of the solvent, precipitation occurs and nuclei are deposited on the anode. Then the above steps proceed continuously. Finally, the polymer films electrodeposit on the surface of the electrode. ${ }^{27}$

To investigate the electropolymerization progress of PEDOT films in the two electrolytes, successive cyclic voltammograms were measured with the scanning rate maintained at $30 \mathrm{mV} \mathrm{s}^{-1}$, as shown in Fig. 2. Although the redox waves are observed in Fig. 2(a) and (b), the better curves with apparent redox waves and increasing intervals of the peak current densities are clearly seen for the electrolyte containing acetonitrile (Fig. 2(b)). This indicated that the PEDOT film with a faster polymerization rate was formed on the electrode surface in the electrolyte containing acetonitrile. The potential shift of the wave current maximum provides information about the increase in the electrical resistance of the PEDOT films.

Fig. 3 shows the relationship between the electropolymerization time and the current density at an applied potential of $1.52 \mathrm{~V}$ for the different electrolytes. In the water/ methanol electrolyte, the current density exhibited a maximum value of $13.4 \mathrm{~A} \mathrm{~m}^{-2}$ at $10 \mathrm{~s}$. Even though a constant potential was applied to the electrolyte, the current density decreased rapidly thereafter until the electropolymerization time reached $120 \mathrm{~s}$. With increasing electropolymerization times, the current density decreased very slowly. The current density at the electropolymerization time of $480 \mathrm{~s}$ was $2.5 \mathrm{~A} \mathrm{~m}^{-2}$,

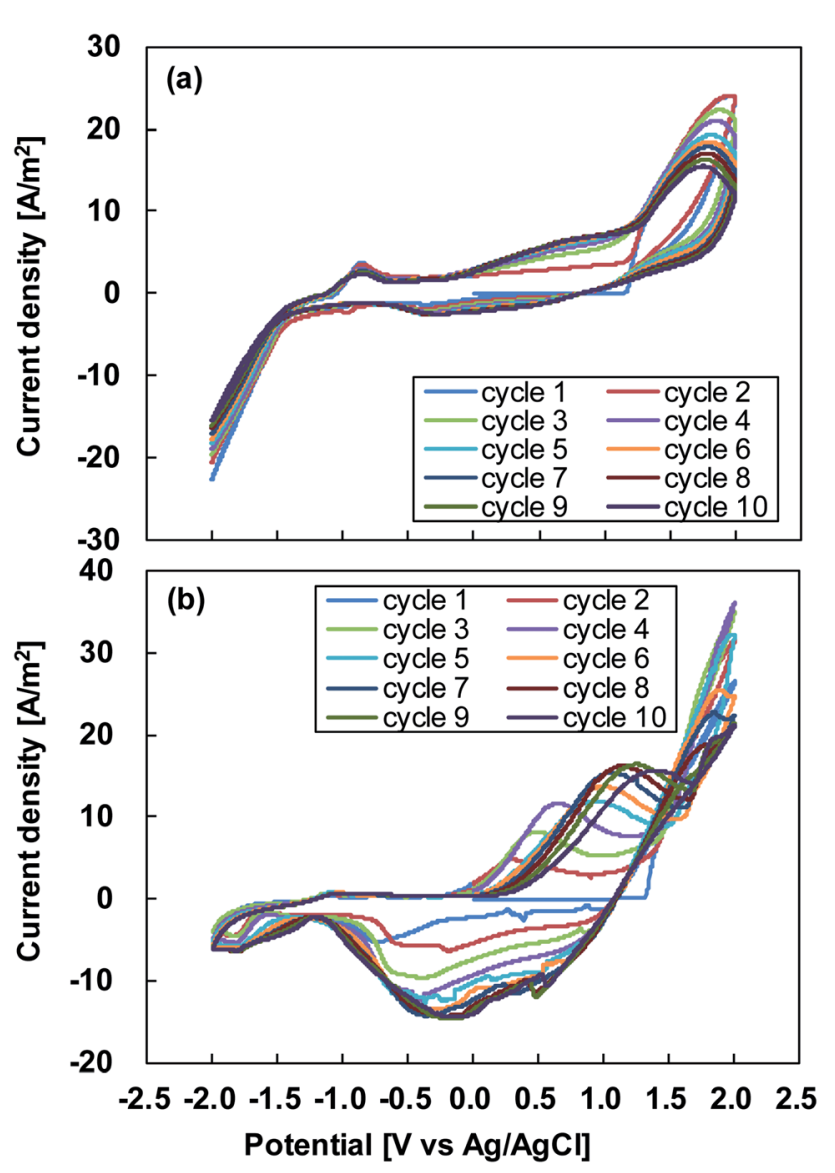

Fig. 2 Successive cyclic voltammograms obtained at a scanning rate of $30 \mathrm{mV} \mathrm{s}^{-1}$ during electropolymerization using (a) water/methanol electrolyte and (b) acetonitrile electrolyte. 


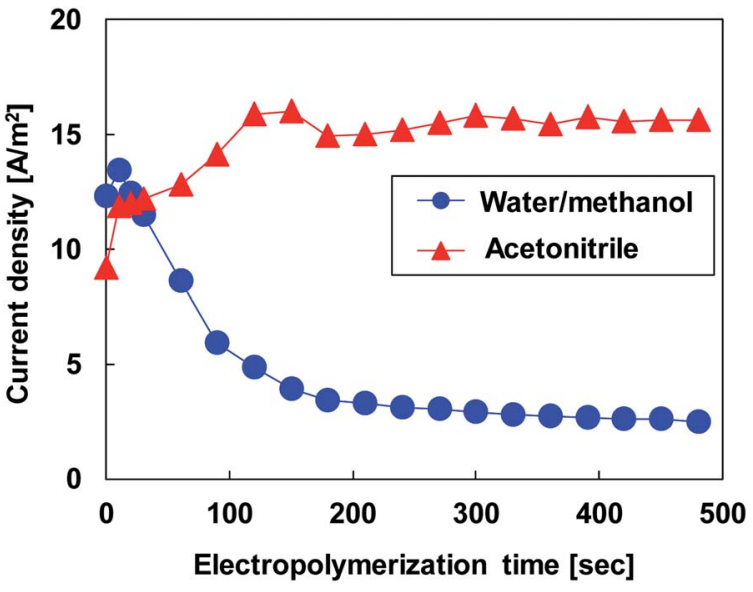

Fig. 3 Relationship between current density and electropolymerization time for different electrolytes.

which was $19 \%$ of the maximum value. This result corresponds with the trend seen in the cyclic voltammogram in Fig. 1(a), which shows passivation of the electrode through nonconductive deposits occurring over longer electropolymerization times. The current density at the beginning of the electropolymerization process $(\sim 1 \mathrm{~s})$ for the acetonitrile electrolyte was $8.6 \mathrm{~A} \mathrm{~m}^{-2}$, which was lower than that for the water/methanol electrolyte at the same point in time. This can be explained by the differences in onset potentials. Higher onset potentials retarded the oxidation of EDOT. The current density rapidly increased as the electropolymerization time increased and rose higher than that of the water/methanol electrolyte at the electropolymerization time of $30 \mathrm{~s}$. The current density increased further when the electropolymerization time reached $120 \mathrm{~s}$. The current density remained almost constant thereafter at $15.7 \mathrm{~A}$ $\mathrm{m}^{-2}$, which was approximately six times higher than that in the water/methanol electrolyte at the same point in time.

\section{Structural properties of PEDOT films}

The relationship between the thickness of PEDOT films and electropolymerization time is shown in Fig. 4. The thickness of the PEDOT films using both electrolytes increased linearly as the electropolymerization time was increased but the increase in ratio, i.e., the deposition rate, of the films produced using acetonitrile was approximately 3.5 times higher than that of the films produced using water/methanol. This trend is supported by the result in Fig. 2. At the electropolymerization time of $480 \mathrm{~s}$, the thicknesses of the thin films produced using water/ methanol and acetonitrile were 5.3 and $18.3 \mu \mathrm{m}$, respectively. This trend therefore indicates that the deposition rate of PEDOT films depends on the oxidation current density. The insets in this figure show photographs of PEDOT films produced with different polymerization times and electrolytes. With increasing film thickness, the film color in both samples became a deep blue, which is the natural color of PEDOT films. ${ }^{44,45}$ The transparency in the film produced using water/methanol was lost at a film thickness of $4.8 \mu \mathrm{m}$ at the electropolymerization time of $180 \mathrm{~s}$; that in the film produced using acetonitrile was lost at

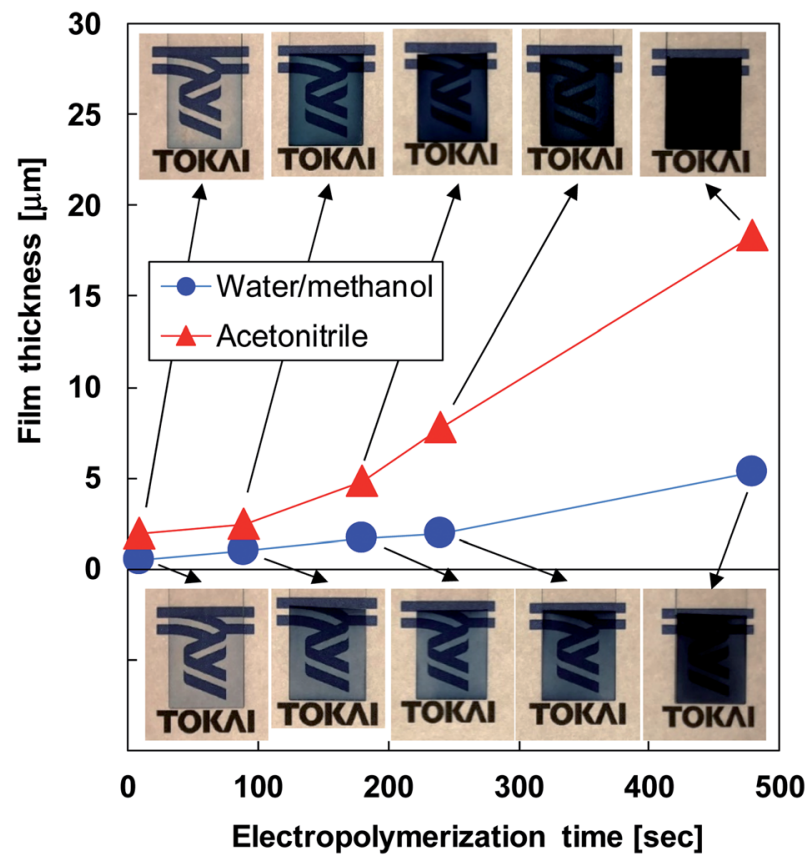

Fig. 4 Relationship between PEDOT film thickness and electropolymerization time for different electrolytes.

a film thickness of $5.3 \mu \mathrm{m}$ at the electropolymerization time of $480 \mathrm{~s}$.

The surface SEM images of PEDOT thin films formed with different electropolymerization time and electrolytes are shown in Fig. 5. EDOT gathered during the short electropolymerization time (10 s) using the water/methanol electrolyte and amorphous polymers began to condense sparsely on the substrate (Fig. 5(a)). When the electropolymerization time was increased to $90 \mathrm{~s}$, clear grains with an approximate size of $50 \mathrm{~nm}$ aggregated on the substrate (Fig. 5(b)). Further increasing the electropolymerization time led to a gradual increase in the size and density of the grains (Fig. 5(c) and (d)). At the electropolymerization time of $480 \mathrm{~s}$, aggregated grains with an approximate size of $100 \mathrm{~nm}$ covered the substrate (Fig. 5(e)).

On the other hand, the initial growth of PEDOT in the acetonitrile electrolyte was largely similar to that in the water/ methanol electrolyte (Fig. 5(f)). However, with increasing electropolymerization time, differences in surface morphologies of the PEDOT films produced using the two electrolytes appeared. In Fig. $5(\mathrm{~g})$, it can be seen that aggregated grains are connected with other aggregated grains through polymeric networks. When the electropolymerization time was increased, the fundamental surface morphologies of the PEDOT films did not change (Fig. $5(\mathrm{~h})-(\mathrm{j}))$.

The difference in surface morphologies of PEDOT films produced using the water/methanol and acetonitrile electrolytes was probably due to the type of solvent in the electrolyte. The water/methanol mixture is a protic solvent, while acetonitrile is an aprotic solvent. Protic solvents can reduce the condensation rate by deactivating the EDOT through hydrogen bonding. The network of intermolecular hydrogen bonds provided a shielding cage around the reactant molecules, 

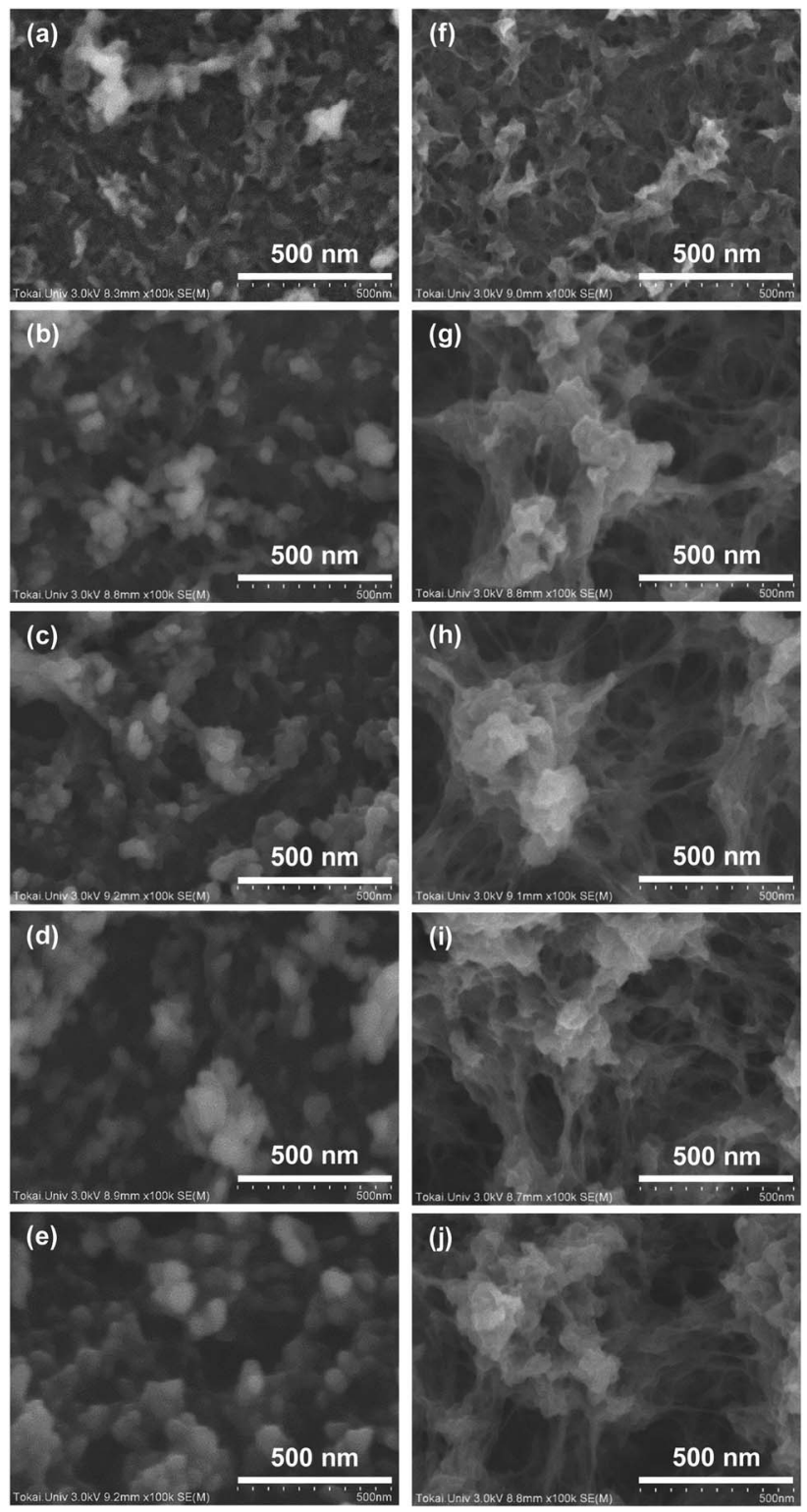

Fig. 5 Surface morphologies of the PEDOT thin films imaged using SEM. Micrographs in (a), (b), (c), (d), and (e) correspond to PEDOT thin films produced using water/methanol electrolyte for electropolymerization times of $10,90,180,240$ and $480 \mathrm{~s}$, respectively. Micrographs in (f), (g), (h), (i), and (j) correspond to PEDOT thin films produced using acetonitrile electrolyte for electropolymerization times of $10,90,180,240$ and 480 s, respectively.

thereby reducing their ability to diffuse and participate in the condensation reaction. ${ }^{46}$ The polymeric networks thus did not develop between the grains. On the other hand, the aprotic solvent did not deactivate the EDOT because it lacked hydrogen atoms capable of forming hydrogen bonds. It could, however, stabilize the reactants with respect to the activated complex. ${ }^{47}$ This led to the polymeric networks connecting the grains.

Fig. 6 shows the FT-IR/ATR spectra of the PEDOT thin films formed using different polymerization times and electrolytes.

In Fig. 6(a), although the signal intensity of the IR spectra of PEDOT films produced using water/methanol increases as the
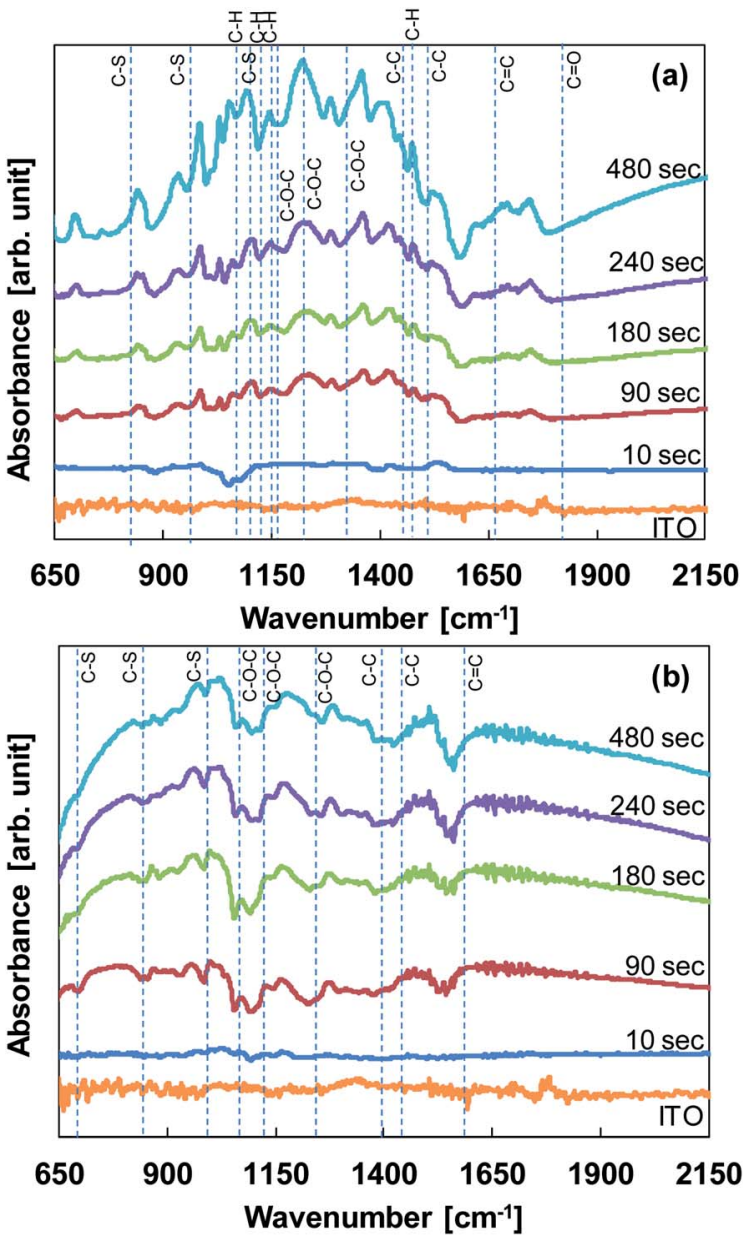

Fig. 6 FT-IR/ATR spectra of PEDOT thin films produced using (a) water/methanol electrolyte and (b) acetonitrile electrolyte.

electropolymerization time increases, the peak positions do not change. The peaks at 1560,1425 , and $1370 \mathrm{~cm}^{-1}$ correspond to $\mathrm{C}=\mathrm{C}$ and $\mathrm{C}-\mathrm{C}$ stretching modes in the thiophene ring. The peaks at 984,845 , and $691 \mathrm{~cm}^{-1}$ can be attributed to the $\mathrm{C}-\mathrm{S}$ bond in the thiophene ring. The peaks at 1231, 1114, and $1050 \mathrm{~cm}^{-1}$ are attributed to $\mathrm{C}-\mathrm{O}-\mathrm{C}$ stretching of the ethylenedioxy group, and the peak at $926 \mathrm{~cm}^{-1}$ corresponds to the ethylenedioxy ring deformation mode. Importantly, peaks suggesting the reaction of hydrogen and PEDOT were observed at $890 \mathrm{~cm}^{-1}$ for the $\mathrm{C}-\mathrm{H}$ bending mode, at 956 and $1378 \mathrm{~cm}^{-1}$ for the $\mathrm{C}-\mathrm{H}$ torsional mode, and at 1020 and $1040 \mathrm{~cm}^{-1}$ for the $\mathrm{C}-\mathrm{H}$ stretching mode. This is because of the protic nature of the solvent. There is evidence indicating that the bonding between PEDOT and hydrogen may hinder film formation, as shown in the SEM images of Fig. 5 .

On the other hand, in Fig. 6(b), the peak positions of the IR spectra in PEDOT films using acetonitrile did not change as the electropolymerization time increased. The typical IR peaks derived from $\mathrm{C}=\mathrm{C}$ and $\mathrm{C}-\mathrm{C}$ stretching modes, the $\mathrm{C}-\mathrm{S}$ bond in the thiophene ring, and the $\mathrm{C}-\mathrm{O}-\mathrm{C}$ stretching were observed, but the $\mathrm{C}-\mathrm{H}$ bending and the $\mathrm{C}-\mathrm{H}$ torsional modes were not observed. 


\section{Thermoelectric properties of PEDOT films}

In this study, the PEDOT films were formed on a conductive ITO substrate. Therefore, to estimate the thermoelectric properties of the PEDOT films alone, it was necessary to eliminate the thermoelectric properties of the ITO substrate from the experimentally measured values using eqn (1) and (2): ${ }^{\mathbf{4 - 4 9}}$

$$
\sigma_{T}=\frac{\sigma_{1} d_{1}+\sigma_{2} d_{2}}{d_{1}+d_{2}}
$$

and

$$
S_{T}=\frac{S_{1} \sigma_{1} d_{1}+S_{2} \sigma_{2} d_{2}}{\sigma_{1} d_{1}+\sigma_{2} d_{2}},
$$

where subscript $T$ indicates the experimentally measured values, which are the combined values of the ITO substrate and PEDOT films, and subscripts 1 and 2 indicate the values of the ITO substrate and PEDOT alone, respectively. The relationship between thermoelectric properties of PEDOT films produced using different electrolytes and electropolymerization times is shown in Fig. 7. Note that the thermoelectric properties of the PEDOT film produced using water/methanol over an electropolymerization time of $480 \mathrm{~s}$ were not measured because the PEDOT film was too fragile.

According to Fig. 7(a), the electrical conductivities of PEDOT films produced using water/methanol and acetonitrile over a short electropolymerization time $(t=10 \mathrm{~s})$ are 705 and $197 \mathrm{~S} \mathrm{~cm}^{-1}$, respectively. With increasing electropolymerization times, the electrical conductivity of PEDOT films produced using both electrolytes decreased. For an electropolymerization time of $240 \mathrm{~s}$, the electrical conductivity values of PEDOT films produced using water/methanol and acetonitrile were 66 and $12 \mathrm{~S} \mathrm{~cm}^{-1}$, respectively. Further increasing the electropolymerization time to $480 \mathrm{~s}$ caused the electrical conductivity of the PEDOT film produced using acetonitrile to become almost identical to that of the film produced at $240 \mathrm{~s}$. These phenomena indicated that the electrical conductivity of PEDOT films decreased as the film thickness increased. A similar trend was also observed in drop-casted PEDOT:PSS films. ${ }^{34,35}$ The inset in Fig. 7(a) shows the relationship between the electrical conductivity of the PEDOT film and film thickness. The electrical conductivity of PEDOT films produced using water/ methanol considerably decreased as the film thickness increased from 0.5 to $1.9 \mu \mathrm{m}$. On the other hand, the electrical conductivity of PEDOT films produced using acetonitrile rapidly decreased as the film thickness increased from 1.9 to $2.4 \mu \mathrm{m}$, and then it decreased slowly when the film thickness increased further. In the film thicknesses ranging from 1.5 to $2.5 \mu \mathrm{m}$ for the PEDOT films produced using both electrolytes, the relationship between the film thickness and electrical conductivity demonstrated a similar trend. It was noted that the two curves (data plots for films produced with water/methanol and acetonitrile) appeared to demonstrate rough continuity. Therefore, it can be concluded that the electrical conductivity of PEDOT films is strongly dependent on the film thickness and less dependent on the type of electrolyte used to produce them, even though the surface morphologies of the PEDOT films differed based on the electrolyte used to produce them. A possible
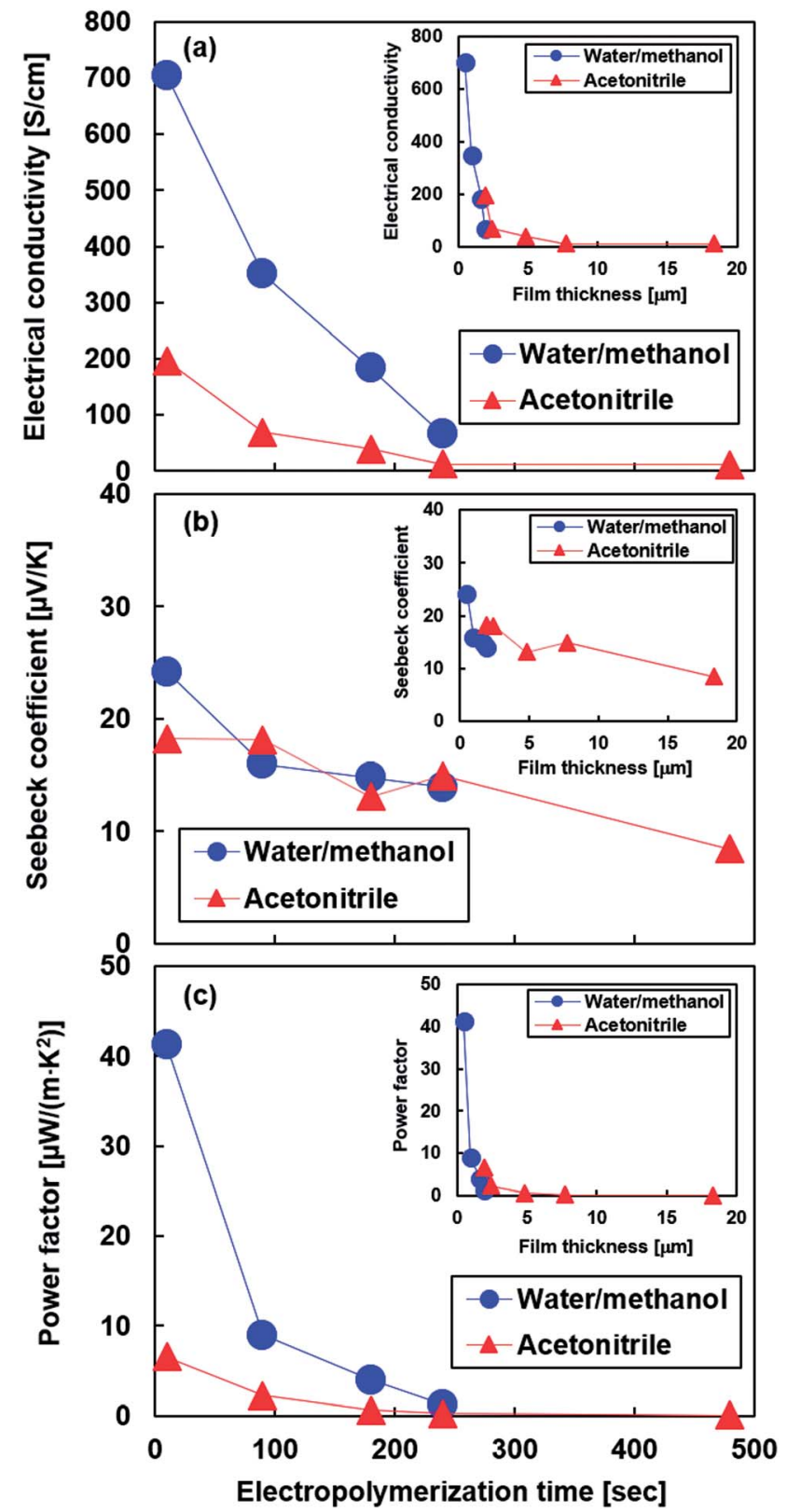

Fig. 7 (a) Electrical conductivity, (b) Seebeck coefficient, and (c) power factor of PEDOT thin films produced in different electrolytes as a function of electropolymerization time. The insets indicate the relationship between the thermoelectric properties and film thickness.

explanation for the decrease in electrical conductivity of PEDOT thin films with increasing thickness is that PEDOT films develop pores or wrinkles due to accumulated strain (Fig. S1 and the text in the $\mathrm{ESI}_{\dagger} \uparrow$ provides more detailed information on this phenomenon). ${ }^{50}$

The Seebeck coefficient as a function of electropolymerization time for PEDOT films produced using water/ methanol and acetonitrile is shown in Fig. 7(b). For short electropolymerization times $(t=10 \mathrm{~s})$, both the PEDOT films produced using water/methanol and those produced using acetonitrile exhibited maximum values of $24.2 \mu \mathrm{V} \mathrm{K}^{-1}$ and 18.2 $\mu \mathrm{V} \mathrm{K}^{-1}$, respectively. The Seebeck coefficient of both the PEDOT 
films decreased as the electropolymerization time increased. The trend in the relationship between the Seebeck coefficient and electropolymerization time was largely identical for PEDOT films produced using both electrolytes. The inset in Fig. 7(b) shows the relationship between the Seebeck coefficient of the PEDOT films and the film thickness. Overall, the Seebeck coefficient of the PEDOT films produced using the two electrolytes decreased as the film thickness increased. However, the decrease in the Seebeck coefficient of the PEDOT film produced using water/methanol was observed in thinner films than that observed in the PEDOT film produced using acetonitrile.

Fig. 7(c) shows the power factor of PEDOT films produced using water/methanol and acetonitrile as a function of the electropolymerization time. The trend in the relationship between the power factor and electropolymerization time was similar to that observed in the relationship between the electrical conductivity and electropolymerization time because the variation in electrical conductivity over time was larger than that of the Seebeck coefficient. For short electropolymerization times $(t=10 \mathrm{~s})$, both the PEDOT films produced using water/ methanol and acetonitrile achieved maximum values of 41.3 $\mu \mathrm{W}\left(\mathrm{m}^{-1} \mathrm{~K}^{-2}\right)$ and $6.5 \mu \mathrm{W}\left(\mathrm{m}^{-1} \mathrm{~K}^{-2}\right)$, respectively. In particular, the performance of the PEDOT film produced using water/ methanol at the electropolymerization time of $10 \mathrm{~s}$ was superior to that of PEDOT films produced using well-established, conventional electropolymerization methods. ${ }^{51,52}$ The power factor of both PEDOT films significantly decreased as the electropolymerization time increased. The inset in Fig. 7(c) shows the relationship between the power factor of PEDOT films and the film thickness. The PEDOT films having higher thicknesses produced using both electrolytes exhibited lower power factors. Therefore, it could be concluded that PEDOT films produced using the water/methanol solvent could achieve a higher thermoelectric performance than PEDOT films produced using acetonitrile. The film thickness is the critical factor in determining the level of thermoelectric performance.

\section{Conclusions}

Electropolymerized PEDOT films were produced using different electrolytes and film thicknesses. The two electrolytes included two different solvents: a water/methanol solvent (protic solvent) and acetonitrile (aprotic solvent), while EDOT and $\mathrm{LiCF}_{3} \mathrm{SO}_{3}$ were commonly included as dopants. Electrochemical analysis of the electrolytes indicated that the polymerization process of EDOT on ITO substrates differed between the two electrolytes. For the electropolymerization of PEDOT films, the electropolymerization time was changed from 10 to $480 \mathrm{~s}$, while the applied voltage was set at $1.52 \mathrm{~V}$. With increasing electropolymerization times, the surface morphology of the PEDOT films produced using water/methanol appeared to contain grains while that of the PEDOT films produced using acetonitrile appeared to contain aggregated grains connected by polymeric networks. The thermoelectric properties were strongly dependent on the film thickness and less dependent on the type of electrolyte used even though there were differences in the surface morphologies.

\section{Author contributions}

Y. S. conceived the experiments, prepared the materials, fabricated the films, and performed electrochemical and SEM measurements. Y. S. and M. T. organized the data and wrote the manuscript. Y. S., M. T., and M. T. oversaw all research phases and revised the manuscript. All authors reviewed and commented on the manuscript.

\section{Conflicts of interest}

The authors declare no competing interests.

\section{Acknowledgements}

This study was partly supported by JSPS KAKENHI Grant Number 16K06752. The authors wish to thank M. Morikawa and Y. Oda at Tokai University for providing support for the experiments.

\section{Notes and references}

1 R. K. Sharma, A. C. Rastogi and S. B. Desu, Pulse polymerized polypyrrole electrodes for high energy density electrochemical supercapacitor, Electrochem. Commun., 2008, 10, 268-272.

2 H. Lee, H. Kim, M. S. Cho, J. Choi and Y. Lee, Fabrication of polypyrrole (PPy)/carbon nanotube (CNT) composite electrode on ceramic fabric for supercapacitor applications, Electrochim. Acta, 2011, 56, 7460-7466.

3 Y. Huang, H. Li, Z. Wang, M. Zhu, Z. Pei, Q. Xue, Y. Huang and C. Zhi, Nanostructured polypyrrole as a flexible electrode material of supercapacitor, Nano Energy, 2016, 22, 422-438.

4 G. Latessa, F. Brunetti, A. Reale, G. Saggio and A. Di Carlo, Piezoresistive behaviour of flexible PEDOT:PSS based sensors, Sens. Actuators, B, 2009, 139, 304-309.

5 Z. Wang, J. Xu, Y. Yao, L. Zhang, Y. Wen, H. Song and D. Zhu, Facile preparation of highly water-stable and flexible PEDOT:PSS organic/inorganic composite materials and their application in electrochemical sensors, Sens. Actuators, B, 2014, 196, 357-369.

6 H. Zhang, J. Xu, Y. Wen, Z. Wang, J. Zhang and W. Ding, Conducting poly(3,4-ethylenedioxythiophene):poly(styrenesulfonate) film electrode with superior long-term electrode stability in water and synergistically enhanced electrocatalytic ability for application in electrochemical sensors, Synth. Met., 2015, 204, 39-47.

7 K. Kawano, J. Sakai, M. Yahiro and C. Adachi, Effect of solvent on fabrication of active layers in organic solar cells based on poly (3-hexylthiophene) and fullerene derivatives, Sol. Energy Mater. Sol. Cells, 2009, 93, 514-518.

8 Y.-M. Xiao, J.-Y. Lin, J.-H. Wu, S.-Y. Tai and G.-T. Yue, Pulse potentiostatic electropolymerization of high performance PEDOT counter electrodes for Pt-free dye-sensitized solar cells, Electrochim. Acta, 2012, 83, 221-226. 
9 Y. Hiroshige, M. Ookawa and N. Toshima, High thermoelectric performance of poly (2, 5dimethoxyphenylenevinylene) and its derivatives, Synth. Met., 2006, 156, 1341-1347.

10 Q. Wei, M. Mukaida, K. Kirihara, Y. Naitoh and T. Ishida, Recent progress on PEDOT-based thermoelectric materials, Materials, 2015, 8, 732-750.

$11 \mathrm{R}$. Yue and J. Xu, Poly (3,4-ethylenedioxythiophene) as promising organic thermoelectric materials: a mini-review, Synth. Met., 2012, 162, 912-917.

12 K. Kato, H. Hagino and K. Miyazaki, Fabrication of bismuth telluride thermoelectric films containing conductive polymers using a printing method, J. Electron. Mater., 2013, 42, 1313-1318.

13 A. Elschner, S. Kirchmeyer, W. Lövenich, U. Merker, and K. Reuter, PEDOT: Principles and Applications of an Intrinsically Conductive Polymer, CRC Press, Boca Raton, 2011, pp. 91-109.

$14 \mathrm{~N}$. Toshima, Conductive polymers as a new type of thermoelectric material, Macromol. Symp., 2002, 186, 81-86.

15 O. Bubnova and X. Crispin, Towards polymer-based organic thermoelectric generators, Energy Environ. Sci., 2012, 5, 9345-9362.

16 H. Yamamuro, N. Hatsuta, M. Wachi, Y. Takei and M. Takashiri, Combination of electrodeposition and transfer processes for flexible thin-film thermoelectric generators, Coatings, 2018, 8, 22.

17 Y. Ito, M. Mizoshiri, M. Mikami, T. Kondo, J. Sakurai and S. Hata, Fabrication of thin-film thermoelectric generators with ball lenses for conversion of near-infrared solar light, Jpn. J. Appl. Phys., 2017, 56, $06 \mathrm{GN} 06$.

18 J. Kurosaki, A. Yamamoto, S. Tanaka, J. Cannon, K. Miyazaki and H. Tsukamoto, Fabrication and evaluation of a thermoelectric microdevice on a free-standing substrate, J. Electron. Mater., 2009, 38, 1326-1330.

$19 \mathrm{H}$. Yamamuro and M. Takashiri, Power generation in slopetype thin-film thermoelectric generators by the simple contact of a heat source, Coatings, 2019, 9, 63.

20 M. Culebras, K. Choi and C. Cho, Recent progress in flexible organic thermoelectrics, Micromachines, 2018, 9, 638.

21 M. C. Morvant and J. R. Reynolds, In situ conductivity studies of poly (3,4-ethylenedioxythiophene), Synth. Met., 1998, 92, 57-61.

22 L. Niu, C. Kvarnström and A. Ivaska, Mixed ion transfer in redox processes of poly(3,4-ethylenedioxythiophene), $J$. Electroanal. Chem., 2004, 569, 151-160.

23 X. Wang, P. Sjöberg-Eerola, J.-E. Eriksson, J. Bobacka and M. Bergelin, The effect of counter ions and substrate material on the growth and morphology of poly(3,4ethylenedioxythiophene) films: towards the application of enzyme electrode construction in biofuel cells, Synth. Met., 2010, 160, 1373-1381.

24 T. Deguchi, H. Tomeoku and M. Takashiri, Preparation and characterization of electropolymerized PEDOT thin films with different dopant anions, Jpn. J. Appl. Phys., 2016, 55, 06GK03.
25 M. Takahashi, T. Deguchi and M. Takashiri, Effect of ultraviolet irradiation on poly (3,4-ethylenedioxythiophene) doped with $\mathrm{ClO}_{4}$ thin films using electropolymerization, Jpn. J. Appl. Phys., 2017, 56, 06GJ03.

26 B. Lu, J. Yan, J. Xu, S. Zhou and X. Hu, Novel electroactive proton-doped conducting poly(aromatic ethers) with good fluorescence properties via electropolymerization, Macromolecules, 2010, 43, 4599-4608.

27 H. Gu, S. Ming, K. Lin, S. Chen, X. Liu, B. Lu and J. Xu, Isoindigo as an electron-deficient unit for highperformance polymeric electrochromics, Electrochim. Acta, 2018, 260, 772-782.

28 X. Chen, K. Z. Xing and O. Inganäs, Electrochemically induced volume changes in poly (3,4ethylenedioxythiophene), Chem. Mater., 1996, 8, 2439-2443.

29 C. Kvarnström, H. Neugebauer, S. Blomquist, H. J. Ahonen, J. Kankare and A. Ivaska, In situ spectroelectrochemical characterization of poly(3,4-ethylenedioxythiophene), Electrochim. Acta, 1999, 44, 2739-2750.

30 V. Seshadri, L. Wu and G. A. Sotzing, Conjugated polymers via electrochemical polymerization of thieno[3,4-b] thiophene (T34bT) and 3,4-ethylenedioxythiophene (EDOT), Langmuir, 2003, 19, 9479-9485.

31 Y. Seki, M. Takahashi and M. Takashiri, Enhanced thermoelectric properties of electropolymerized poly $(3,4-$ ethylenedioxythiophene) thin films by optimizing electrolyte temperature and thermal annealing temperature, Org. Electron., 2018, 55, 112-116.

32 X. J. Wang and K. Y. Wong, Effects of a base coating used for electropolymerization of poly (3,4-ethylenedioxythiophene) on indium tin oxide electrode, Thin Solid Films, 2006, 515, 1573-1578.

33 N. Sakmeche, J. J. Aaron, M. Fall, S. Aeiyach, M. Jouini, J. C. Lacroix and P. C. Lacaze, Anionic micelles; a new aqueous medium for electropolymerization of poly $(3,4-$ ethylenedioxythiophene) films on Pt electrodes, Chem. Commun., 1996, 24, 2723-2724.

34 A. Lima, P. Schottland, S. Sadki and C. Chevrot, Electropolymerization of 3,4-ethylenedioxythiophene and 3,4-ethylenedioxythiophene methanol in the presence of dodecylbenzenesulfonate, Synth. Met., 1998, 93, 33-41.

35 W. J. Doherty, R. J. Wysocki, N. R. Armstrong and S. Saavedra, Electrochemical copolymerization and spectroelectrochemical characterization of 3,4ethylenedioxythiophene and 3,4-ethylenedioxythiophenemethanol copolymers on indium-tin oxide, Macromolecules, 2006, 39, 4418-4424.

36 V. Linseis, Z. M. Hassan, H. Reith, J. Garcia, K. Nielsch, H. Baumgart, E. Redel and P. Woias, Complete thermoelectric characterization of PEDOT:PSS thin films with a novel ZT test chip platform, Phys. Status Solidi A, 2018, 215, 1700930.

37 H. Anno, T. Nishinaka, M. Hokazono, N. Oshima and N. Toshima, Thermoelectric power-generation characteristics of PEDOT:PSS thin-film devices with different thicknesses on polyimide substrates, J. Electron. Mater., 2015, 44, 2105-2112. 
38 N. Hatsuta, D. Takemori and M. Takashiri, Effect of thermal annealing on the structural and thermoelectric properties of electrodeposited antimony telluride thin films, J. Alloys Compd., 2015, 685, 147-152.

39 Y. Li, Y. Cao, J. Gao, D. Wang, G. Yu and A. J. Heeger, Electrochemical properties of luminescent polymers and polymer light-emitting electrochemical cells, Synth. Met., 1999, 99, 243-248.

40 N. A. Zubair, N. A. Rahman, H. N. Lim and Y. Sulaiman, Production of conductive PEDOT-coated PVA-GO composite nanofibers, Nanoscale Res. Lett., 2017, 12, 113.

41 K. Krukiewicz, T. Jarosz, A. P. Herman, R. Turczyn, S. Boncel and J. K. Zak, Synth. Met., 2016, 217, 231-236.

42 E. Poverenov, M. Li, A. Bitler and M. Bendikov, Major effect of electropolymerization solvent on morphology and electrochromic properties of PEDOT films, Chem. Mater., 2010, 22, 4019-4025.

$43 \mathrm{M}$. Zhou and J. Heinze, Electropolymerization of pyrrole and electrochemical study of polypyrrole. 3. nature of "water effect" in acetonitrile, J. Phys. Chem. B, 1999, 103, 8451-8457.

44 Y. Zhu, M. T. Otley, A. Kumar, M. Li, X. Zhang, C. Asemota and G. A. Sotzing, Neutral color tuning of polymer electrochromic devices using an organic dye, Chem. Commun., 2014, 50, 8167-8170.

45 J.-H. Kang, S.-M. Paek, Y. B. Choy, S.-J. Hwang and J.-H. Choy, Enhanced contrast of electrochromic full cell systems with nanocrystalline PEDOT-Prussian blue, $J$. Nanosci. Nanotechnol., 2007, 7, 4131-4134.
46 I. Artaki, T. W. Zerda and J. Jonas, Solvent effects on the condensation stage of the sol-gel process, J. Non-Cryst. Solids, 1986, 81, 381-395.

47 C.-N. Liao, T.-H. She, P.-J. Liao and H.-S. Chu, Oscillatory transport properties of thermally annealed $\mathrm{Bi} / \mathrm{Te}$ multilayer thin films, J. Electrochem. Soc., 2007, 154, H304-H308.

48 Y. Sasaki and M. Takashiri, Effects of Cr interlayer thickness on adhesive, structural, and thermoelectric properties of antimony telluride thin films deposited by radio-frequency magnetron sputtering, Thin Solid Films, 2016, 619, 195-201.

49 M. Takashiri, T. Makioka and H. Yamamuro, Promotion of crystal growth in as-grown $\mathrm{Bi}_{2} \mathrm{Te}_{3}$ electrodeposited films without micro-pores using sputtered $\mathrm{Bi}_{2} \mathrm{Te}_{3}$ seed layers deposited on a glass substrate, J. Alloys Compd., 2018, 764, 802-808.

50 J. Y. Oh, M. Shin, J. B. Lee, J.-H. Ahn, H. K. Baik and U. Jeong, Effect of PEDOT nanoffbril networks on the conductivity, flexibility, and coatability of PEDOT:PSS films, ACS Appl. Mater. Interfaces, 2014, 6, 6954-6961.

51 H. Gu, S. Ming, K. Lin, H. Liu, S. Chen, B. Lu and J. Xu, Thermoelectric properties of poly(selenophene-co-3, 4ethylenedioxythiophene) via electropolymerization, $J$. Electron. Mater., 2017, 46, 3124-3130.

52 L. Zhang, T. Goto, I. Imae, Y. Sakurai and Y. Harima, Thermoelectric properties of PEDOT films prepared by electrochemical polymerization, J. Polym. Sci., Part B: Polym. Phys., 2017, 55, 524-531. 